\title{
CONCENTRAÇÃO E CONTEÚDO DE MACRONUTRIENTES DA BIOMASSA DE MIMOSA SCABRELLA BENTHAM, EM DIFERENTES CLASSES DE SÍTIO, IDADE E DIÂMETRO
}

\section{CONCENTRATION AND CONTENT OF MACRONUTRIENT IN THE MIMOSA SCABRELLA BENTHAM BIOMASS, AT DIFFERENT SITE, AGE AND DIAMETER CLASSES}

\author{
Saulo Jorge TÉO ${ }^{1}$ \\ Sebastião do Amaral MACHADO ${ }^{2,4}$ \\ Carlos Bruno REISSMANN ${ }^{3,4}$ \\ Afonso FIGUEIREDO FILHO ${ }^{2,4}$
}

\begin{abstract}
RESUMO
Os objetivos deste trabalho foram quantificar e avaliar as concentrações e conteúdos de macronutrientes na biomassa aérea de bracatinga (Mimosa scabrella Bentham) sob diferentes classes de sítio, idade e diâmetro. Ao todo, 25 árvores foram amostradas em diferentes bracatingais da região metropolitana de Curitiba, no Estado do Paraná. No campo, as árvores foram medidas e separadas nos seguintes compartimentos da biomassa: folhas, galhos $<4 \mathrm{~cm}$, galhos $\geq 4 \mathrm{~cm}$, madeira e casca do fuste. Posteriormente, amostras foram levadas ao laboratório para determinação da massa seca e macronutrientes. O efeito das classes de sítio, idade e diâmetro foram avaliados por meio de delineamentos estatísticos inteiramente casualizados. Os resultados encontrados indicaram que, as concentrações dos macronutrientes por compartimentos da biomassa apresentaram a seguinte ordem decrescente: folhas > casca > galhos $>$ madeira. $\mathrm{O}$ compartimento da biomassa que apresentou os maiores conteúdos de macronutrientes foi a madeira. De acordo com os resultados, as concentrações de macronutrientes na biomassa aérea de bracatinga não apresentaram relação direta com as classes de idade e diâmetro e somente as concentrações de $\mathrm{P}$ e $\mathrm{K}$ nas folhas diminuíram da melhor para a pior classe de sítio. Não foram observadas relações de aumento ou diminuição entre os conteúdos de macronutrientes da biomassa de bracatinga e as diferentes classes de sítio e idade, entretanto, estes foram diretamente proporcionais aos diâmetros das árvores.

Palavras-chave: Macronutrientes; ciclagem de nutrientes; biomassa; bracatinga.
\end{abstract}

\begin{abstract}
The aims of this work were to quantify and to evaluate macronutrient concentration and content of the Mimosa scabrella Bentham above-ground biomass under different site, age and diameter classes. Twenty five trees were sampled in several stands of the Curitiba metropolitan region, Paraná State, Brazil. In the field, the trees were measured and separated in leaves, twigs, branches, stem wood and bark. Later on, a fraction of each tree component was carried to the laboratory for the determination of dry weight and macronutrient. The site, age and diameter class effects were evaluated by completely random statistic design. The results showed that, the macronutrient concentration by compartment of biomass presented the following decreasing order: leaves $>$ stem bark $>$ twigs $>$ branches $>$ stem wood. The macronutrient content in the stem wood presented the highest quantity. According to the results, the macronutrient concentrations of the Mimosa scabrella above-ground biomass didn't show any relationship with the age and diameter classes, on the other hand, $\mathrm{P}$ and $\mathrm{K}$ leaf concentrations increased with site quality. There was no relationship between macronutrient content of the Mimosa scabrella above-ground biomass, site and age classes. Nevertheless, diameter classes presented a significant and coherent influence over macronutrient content, which increases with the diameter classes.
\end{abstract}

Key-words: Macronutrients; nutrient cycling; biomass.

\footnotetext{
${ }^{1}$ Mestre em Engenharia Florestal, Professor do curso de Engenharia Florestal da Unoesc - Campus de Xanxerê, Rua Dirceu Giordani, 696, Mestre em Engenharia Florestal, Professor do curso de Engenharia Florestal da Unoesc - Campus de Xanxerê,
Jardim Universitário, 89820-000, Xanxerê, SC, Brasil. E-mail: sauloteo@yahoo.com.br. Autor para correspondência.

2 Dr. em Ciências Florestais, Departamento de Ciências Florestais da Universidade Federal do Paraná, Curitiba, PR, Brasil. E-mail: samachado@ufpr.br; afig@ufpr.br

${ }^{3}$ Dr. em Ciências Florestais, Departamento de Solos e Engenharia Agrícola da Universidade Federal do Paraná, Curitiba, PR, Brasil. E-mail: reissman@ufpr.br

${ }^{4}$ Pesquisador do CNPq.
} 
TÉO, S. J. et al. Concentração e conteúdo de macronutrientes...

\section{INTRODUÇÃO}

A bracatinga (Mimosa scabrella Bentham) é uma espécie comum na região metropolitana de Curitiba, de grande importância econômica, social e ambiental. É manejada sob um sistema agroflorestal tradicional, desde o início do século 20, o qual prevê a regeneração do bracatingal induzida pelo fogo, a associação ao cultivo de milho e feijão no primeiro ano de rotação, seguindo-se o raleio do bracatingal durante a colheita das culturas agrícolas, corte raso aproximadamente aos 7 anos de idade, utilizando sistemas de colheita sem mecanização e com a utilização de mão-de-obra familiar.

Somarriba \& Kass (2001), baseados em extensa revisão bibliográfica, reportaram que os solos dos bracatingais quase invariavelmente são de baixa fertilidade, com 6 a $8 \%$ de matéria orgânica $(0-20 \mathrm{~cm}$ de profundidade), com valores de $\mathrm{pH}$ frequentemente menores do que 4 , níveis de fósforo abaixo de $10 \mathrm{mg} \mathrm{kg}^{-1}$, soma de bases abaixo de $1 \mathrm{cmol}_{\mathrm{c}} \mathrm{kg}^{-1}$ e níveis de alumínio trocável acima de $5 \mathrm{cmol}_{\mathrm{c}} \mathrm{kg}^{-1}$. A maioria dos solos associados aos bracatingais é classificada como Cambissolos Álicos, variando de profundo a pouco profundo, principalmente em relevo forte ondulado.

O sistema agroflorestal da bracatinga implica em grande exportação de nutrientes pela colheita de madeira, de grãos das culturas agrícolas associadas, e da queima de resíduos pósexploração, que visa à regeneração do bracatingal. Além disso, em muitos casos, o número de rotações em um mesmo terreno é elevado e a adubação de reposição não é aplicada, causando decréscimos de produtividade (Baggio \& Carpanezzi, 1997). Todos esses fatores tornam ainda mais importante o conhecimento do comportamento dos nutrientes nos diferentes compartimentos da biomassa da bracatinga, em diferentes classes de sítio, idade e diâmetro.

Alguns autores relataram efeitos significativos da idade sobre as concentrações de nutrientes nos diferentes compartimentos da biomassa de diferentes espécies. De maneira geral, há uma diminuição da concentração de nutrientes com o aumento da idade (Pereira et al., 1984; Ranger et al., 1995; Laclau et al.; 2000). Estes resultados podem ser explicados pelo fato de que quanto maior é a idade da árvore, geralmente, menores são as proporções de tecidos fisiologicamente ativos da planta.

Essa mesma tendência de diminuição das concentrações de nutrientes é encontrada com relação ao diâmetro das árvores, e pode ser explicada pela alta correlação entre diâmetro e idade.

Com relação aos conteúdos de nutrientes, Singh (1982) reportou que o conteúdo de nutrientes de um povoamento florestal varia substancialmente de acordo com o grau de absorção de nutrientes e acúmulo de biomassa, os quais são afetados pela idade das árvores e pela qualidade de sítio, mesmo dentro de uma mesma espécie.
Várias pesquisas relataram uma relação diretamente proporcional entre o conteúdo de nutrientes, idade e o diâmetro. Segundo Singh (1982), esse padrão ocorreu para Pinus patula em todos os nutrientes, porque a variação da massa seca com a idade é muito maior do que a variação das concentrações de qualquer um dos nutrientes.

Estudos com diferentes espécies de eucalipto relataram que, de maneira geral, houve um aumento do conteúdo de nutrientes na biomassa com relação à idade (Bellote et al., 1983; Pereira et al., 1984; Laclau et al., 2000).

O objetivo desta pesquisa foi avaliar os efeitos de diferentes classes de sítio, idade e diâmetro sobre as concentrações e conteúdos de macronutrientes nos diferentes compartimentos da biomassa de bracatinga, na região metropolitana de Curitiba, Estado do Paraná.

\section{MATERIAL E MÉTODOS}

A área de estudo abrangeu 7 povoamentos naturais de bracatinga localizados em pequenas propriedades rurais situadas nos municípios de Almirante Tamandaré, Campina Grande do Sul, Campo Magro e Colombo, na porção norte da região metropolitana de Curitiba.

O clima da região é o Cfb da classificação de Köeppen, com temperatura média anual de 16,5 ${ }^{\circ} \mathrm{C}$, precipitação média anual de $1500 \mathrm{~mm}$, o mês mais rico em chuva é janeiro e o mais pobre agosto (Maack, 2002). Em média, a altitude da área de estudo é de $950 \mathrm{~m}$ acima do nível do mar.

Os bracatingais amostrados foram selecionados visando cobrir toda a variação da qualidade de sítios, com base no trabalho de Machado et al. (1997), e também, diferentes classes de diâmetro. A classificação de sítios realizada por esses autores baseou-se no desenvolvimento da altura dominante dos bracatingais da região metropolitana de Curitiba, de modo que, quanto maior a altura das árvores dominantes, melhor é a qualidade de sítio e produtividade dos bracatingais.

Ao todo, foram amostradas 25 árvores, das diversas localidades distribuídas pela área de estudo. Os trabalhos de campo foram concentrados nos meses de junho e julho de 2007. Marcou-se e mediu-se o diâmetro à altura do peito (DAP) das árvores, as quais posteriormente, foram derrubadas e separadas nos seguintes compartimentos da biomassa aérea: folhas, galhos menores do que 4 $\mathrm{cm}$ de diâmetro, galhos maiores ou iguais a $4 \mathrm{~cm}$ de diâmetro, madeira do fuste e casca do fuste.

A massa verde de cada compartimento foi determinada no campo e uma amostra de cada um deles foi retirada e embalada em sacos plásticos para serem transportadas até o laboratório. No fuste, foram retiradas 3 amostras: uma a $50 \mathrm{~cm}$ da base do fuste, uma exatamente na metade da altura do fuste da árvore, e uma a $50 \mathrm{~cm}$ abaixo do topo do fuste, ou ponto de inversão morfológico. As amostras consistiam em discos de $5 \mathrm{~cm}$ de espessura, em média. As amostras dos galhos, 
TÉO, S. J. et al. Concentração e conteúdo de macronutrientes...

tanto maiores ou iguais, como menores do que 4 $\mathrm{cm}$ de diâmetro foram retiradas abrangendo galhos finos, médios e grossos dentro das suas classes pré -estabelecidas. Por fim, foram retiradas amostras das folhas com aproximadamente $150 \mathrm{~g}$ de massa fresca.

As árvores amostradas apresentaram diâmetro à altura do peito (DAP) médio de $11,2 \mathrm{~cm}$, variando de 7,0 a $20,0 \mathrm{~cm}$; altura total média de $14,3 \mathrm{~m}$, variando de 10,2 a $19,5 \mathrm{~m}$; e idade entre 6 e 14 anos. Das 25 árvores amostradas, 6 estavam na classe de sítio I, 6 na classe de sítio II, 7 na classe de sítio III e 6 na classe de sítio IV. Quanto às classes de idade, 10 árvores tinham idades menores do que 8 anos, 5 árvores tinham idades entre 8 e 9,9 anos, 6 árvores tinham idades entre 10 e 11,9 anos e 4 árvores tinham idades maiores do que 12 anos. As árvores amostradas foram distribuídas em classes diamétricas com intervalos de $3 \mathrm{~cm}$, sendo que 10 árvores possuíam diâmetros menores do que $10 \mathrm{~cm}, 7$ árvores possuíam diâmetros entre 10 e $12,9 \mathrm{~cm}$ e 8 árvores possuíam diâmetros maiores ou iguais a $13 \mathrm{~cm}$.

Em laboratório, as amostras foram picadas e levadas à câmara de secagem a $65^{\circ} \mathrm{C}$ até massa constante para obtenção da massa seca. Posteriormente, elas foram moídas em moinho de facas com peneira de malha 30 mesh $(0,3 \mathrm{~mm})$, e uma alíquota de aproximadamente $15 \mathrm{~g}$ foi retirada e acondicionada em frascos plásticos herméticos, para realização das análises químicas dos macronutrientes.

As análises dos nutrientes $\mathrm{N}, \mathrm{P}, \mathrm{K}, \mathrm{Ca}, \mathrm{Mg}$ foram feitas no Laboratório de Biogeoquímica e Nutrição de Plantas do Departamento de Solos e Engenharia Agrícola do Setor de Ciências Agrárias da Universidade Federal do Paraná.

O N foi determinado após digestão sulfúrica conforme Kjeldahl, utilizando-se um destilador e posterior titulação das amostras seguindo a marcha analítica descrita por Martins \& Reissmann (2007). Após a digestão via seca, em mufla a $500{ }^{\circ} \mathrm{C}$ e solubilização em $\mathrm{HCl} 3 \mathrm{~mol} \mathrm{dm}^{-3}$ (Martins \& Reissmann, 2007), o P foi determinado por colorimetria com vanadato-molibidato de amônio, o K por fotometria de chama, e $\mathrm{Ca}$ e $\mathrm{Mg}$ foram determinados por espectrofotometria de absorção atômica.

Após o cálculo das concentrações e dos conteúdos de macronutrientes para cada um dos compartimentos da biomassa, foi realizada a análise estatística com o propósito de identificar possíveis efeitos das classes de sítio, idade e diâmetro sobre as concentrações e conteúdos de macronutrientes. As árvores do banco de dados foram separadas em diferentes classes de sítio, classes de idade e de diâmetro, as quais constituíram os tratamentos de três delineamentos inteiramente casualizados. O número de árvores em cada tratamento (classe de sítio, de idade e diâmetro) constituíram as repetições dos respectivos delineamentos estatísticos.

As concentrações e conteúdos de macronutrientes em cada compartimento da biomassa constituíram as diferentes variáveis. Ao todo foram analisadas concentrações e conteúdos de 5 macronutrientes para cada um dos 5 compartimentos da biomassa.

A análise estatística iniciou-se com o teste de Bartlett, para verificação da homogeneidade das variâncias dos tratamentos, posteriormente a análise de variâncias e, se necessário, teste de comparação de médias de Tukey $(\alpha=0,05)$.

\section{RESULTADOS E DISCUSSÃO}

$\mathrm{Na}$ Tabela 1 são apresentadas as concentrações e conteúdos médios de macronutrientes nos diferentes compartimentos da biomassa aérea de bracatinga, calculados com base nas 25 árvores do banco de dados.

TABELA 1 - Concentração e conteúdo médio de macronutrientes por compartimento da biomassa aérea de bracatinga, na região metropolitana de Curitiba.

\begin{tabular}{ccccccc}
\hline \multicolumn{7}{c}{ Concentração $\left(\mathrm{g} \mathrm{kg}^{-1}\right)$} \\
\cline { 1 - 5 } Nutrientes & \multirow{2}{*}{ Total } \\
\cline { 2 - 6 } & Folha & Galhos $<4^{*}$ & Galhos $\geq 4^{*}$ & Madeira & Casca & \\
\hline $\mathrm{N}$ & 25,01 & 12,81 & 8,27 & 2,06 & 15,44 & \\
$\mathrm{P}$ & 1,47 & 0,26 & 0,17 & 0,09 & 0,32 & \\
$\mathrm{~K}$ & 4,96 & 3,31 & 3,74 & 2,50 & 4,87 & \\
$\mathrm{Ca}$ & 6,47 & 5,65 & 3,73 & 1,07 & 12,56 & \\
$\mathrm{Mg}$ & 1,78 & 0,50 & 0,42 & 0,20 & 0,62 & \\
\hline \multicolumn{7}{c}{ Conteúdo $\left(\mathrm{g} \mathrm{planta}^{-1}\right)$} \\
$\mathrm{N}$ & 63,47 & 66,86 & 60,58 & 75,90 & 86,05 & 343,18 \\
$\mathrm{P}$ & 3,65 & 1,38 & 1,17 & 3,33 & 1,73 & 11,07 \\
$\mathrm{~K}$ & 12,47 & 17,63 & 23,95 & 100,76 & 27,60 & 178,58 \\
$\mathrm{Ca}$ & 14,63 & 28,17 & 27,58 & 40,20 & 67,92 & 174,08 \\
$\mathrm{Mg}$ & 4,00 & 2,72 & 3,04 & 7,79 & 3,24 & 20,30 \\
\hline
\end{tabular}

\footnotetext{
* diâmetro dos galhos na ponta fina, em cm.
} 
TÉO, S. J. et al. Concentração e conteúdo de macronutrientes...

Todos os macronutrientes, exceto o $\mathrm{Ca}$ apresentaram maiores concentrações nas folhas, seguida da casca, galhos e por último, madeira. Para o $\mathrm{Ca}$ a ordem de concentração relativa aos compartimentos da biomassa foi diferente: casca $>$ folhas > galhos > madeira. Segundo Epstein \& Bloom (2006), o cálcio tende a se tornar imobilizado em tecidos mais velhos e não é deslocado em quantidade para regiões mais jovens, que crescem ativamente. Além disso, o cálcio é um dos elementos responsáveis pelas características físicas da parede celular e tende a se concentrar na casca também em outras espécies florestais, conforme mostram os resultados encontrados em diversos estudos, como Caldeira et al. (2000a), Caldeira et a. (2000b), Caldeira et al. (2001) e Moura et al. (2006) para Acacia mearnsii e Mimosa caesalpiniaefolia, espécies leguminosas, tal como a bracatinga. É possível ainda, que boa parte do $\mathrm{Ca}$ na casca da bracatinga esteja na forma de oxalato, resultante da eliminação do excesso tóxico deste composto e, servindo ao mesmo tempo, de proteção contra herbivoria (Navarro et al., 2007; Prata \& Mendonça, 2009).

\section{Considerando}

os

diferentes

compartimentos da biomassa, o nutriente que apresentou as maiores concentrações foi o $\mathrm{N}$, seguido do $\mathrm{Ca}, \mathrm{K}, \mathrm{Mg}$ e por último, $\mathrm{P}$. No entanto, para os galhos $\geq 4 \mathrm{~cm}$ e para madeira, $\circ \mathrm{K}$ apresentou concentrações maiores, em relação ao $\mathrm{Ca} e$ até mesmo para o $\mathrm{N}$. As grandes concentrações de nitrogênio na biomassa aérea da bracatinga podem ser explicadas pela grande importância deste elemento, como constituinte de compostos carbônicos, de todos os aminoácidos, amidas, proteínas, ácidos nucléicos, nucleotídeos, poliaminas e vários outros tipos de entidades metabólicas (Epstein \& Bloom, 2006). Já, as grandes concentrações de $\mathrm{K}$ na madeira podem estar relacionadas com uma reserva deste elemento nos tecidos parenquimáticos do alburno, que, de acordo com Fabrowski et al. (2005), podem ocupar até $35 \%$ da seção transversal do fuste.

Baggio \& Carpanezzi (1997) encontraram uma ordem semelhante das concentrações dos nutrientes para todos os compartimentos da biomassa de bracatinga, porém foi verificada a predominância do $\mathrm{K}$ sobre o $\mathrm{Ca}$.

As concentrações de $\mathrm{N}, \mathrm{P}$ e $\mathrm{Mg}$ foram semelhantes às encontradas em outros estudos, tanto para bracatinga como para outras espécies leguminosas. Contudo, as concentrações de $\mathrm{K}$ se mostram sempre mais baixas quando comparadas com outros estudos, principalmente para a concentração de $\mathrm{K}$ nas folhas, a qual, de acordo com Perry (1994), está dentro da zona de deficiência para folhagem de espécies florestais. Já as concentrações de $\mathrm{Ca}$ foram sempre mais altas quando comparadas com os trabalhos de Baggio \& Carpanezzi (1997), Caldeira et al. (2000a) e Caldeira et al. (2001), em todos os compartimentos da biomassa, principalmente na casca e nas folhas.

Para o conteúdo total de macronutrientes na biomassa aérea de bracatinga, a seguinte ordem decrescente foi verificada: $\mathrm{N}>\mathrm{K}>\mathrm{Ca}>\mathrm{Mg}>\mathrm{P}$. No caso dos conteúdos de nutrientes, o $\mathrm{K}$ apresentou maiores valores do que o $\mathrm{Ca}$ devido à maiores concentrações em compartimentos que apresentam maior biomassa. Esses resultados são compatíveis com os encontrados por Baggio \& Carpanezzi (1997), para bracatinga na região metropolitana de Curitiba. No entanto, Schumacher \& Caldeira (2001) e Schumacher et al. (2008), trabalhando respectivamente com Eucalyptus globulus e Hovenia dulcis, encontraram maiores quantidades de cálcio na biomassa do que de potássio e, até mesmo, de nitrogênio. Essa diferença pode ser justificada pela capacidade que a bracatinga possui de fixar nitrogênio por simbiose com microrganismos de vida livre no solo, cujos mais comuns são as bactérias do solo, coletivamente chamadas de rizóbios (Epstein \& Bloom, 2006).

Uma informação mais relevante do que as quantidades absolutas de macronutrientes em cada compartimento da biomassa é a participação de cada um desses compartimentos com relação à quantidade total de macronutrientes. A Figura 1 mostra as porcentagens de macronutrientes que cada compartimento da biomassa contém, considerando uma média para todos os macronutrientes.

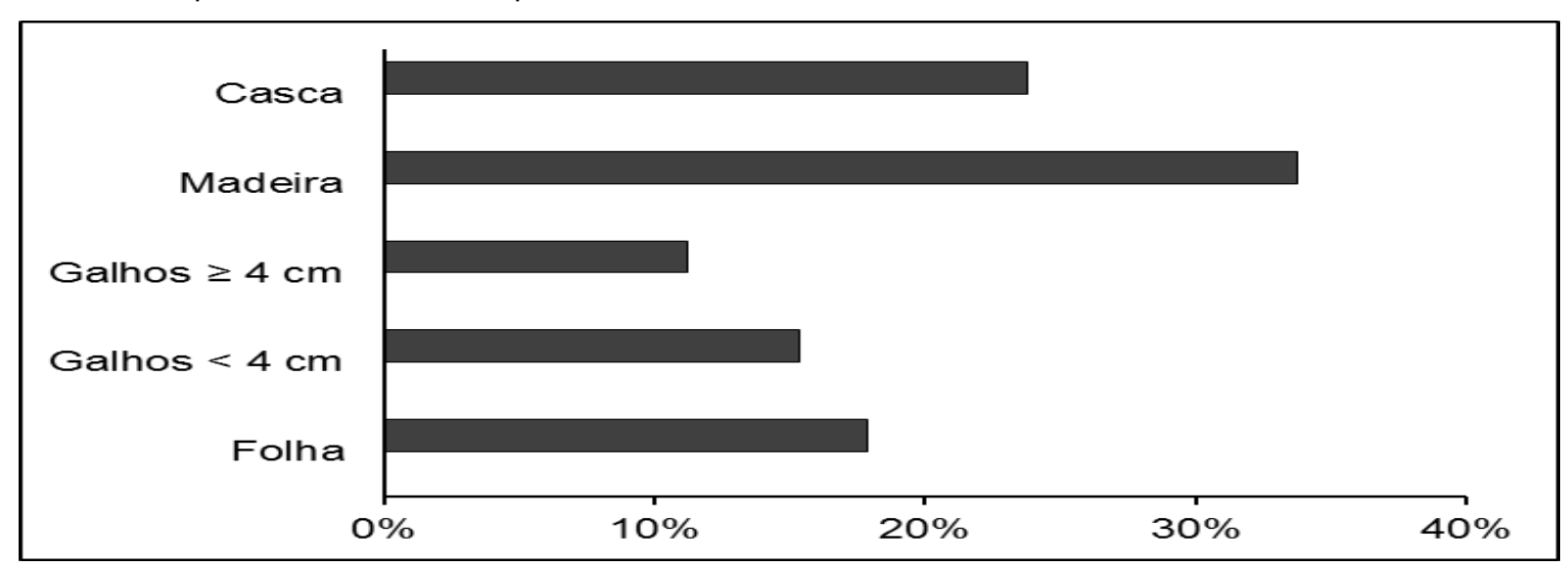

FIGURA 1 - Proporção média de macronutrientes acumulados por compartimento da biomassa aérea de bracatinga, em porcentagem com relação ao conteúdo total de macronutrientes. 
TÉO, S. J. et al. Concentração e conteúdo de macronutrientes...

A madeira foi o compartimento da biomassa que apresentou as menores concentrações de macronutrientes. Todavia, por possuir os maiores valores para massa seca, que, por sua vez, influenciam diretamente no cálculo dos conteúdos de nutrientes, este foi o compartimento que apresentou a maior porcentagem de macronutrientes, com $33,8 \%$, seguido da casca, folhas, galhos $<4 \mathrm{~cm}$ e galhos $\geq 4 \mathrm{~cm}$, com $23,8 \%$, $17,9 \%, 15,3 \%$ e $11,2 \%$, respectivamente.

Ao se distinguir fuste (madeira e casca) e copa (galhos e folhas), o fuste apresenta, em média, $57,6 \%$ dos macronutrientes acumulados na biomassa aérea de bracatinga, enquanto a copa $44,4 \%$. Tradicionalmente, os galhos da bracatinga, até o diâmetro de cerca de $4 \mathrm{~cm}$, também são utilizados como lenha. Dessa maneira, a lenha (madeira, casca e galhos maiores ou iguais a $4 \mathrm{~cm}$ ) da bracatinga acumula $68,8 \%$ dos macronutrientes da biomassa aérea.
Baggio \& Carpanezzi (1997) relataram que $71,3 \%$ do conteúdo total de nutrientes (macro e micronutrientes) está na lenha de bracatinga, muito próximo do valor encontrado neste estudo, para macronutrientes. Contudo, esses autores consideraram a lenha como sendo o material lenhoso com casca, de diâmetros iguais ou maiores que $3 \mathrm{~cm}$, e não $4 \mathrm{~cm}$ como neste trabalho.

O tipo de exploração da madeira é determinante para a exportação de nutrientes, conforme reportado por Caldeira et al. (2002), para Acacia mearnsii, no município de Butiá - RS. De acordo com esses autores, se a colheita da madeira fosse realizada sem casca, a exportação de $\mathrm{N}$ diminuiria em duas vezes e de $\mathrm{Ca}$, em mais de três vezes.

$\mathrm{Na}$ Tabela 2 são apresentadas as concentrações $\left(\mathrm{g} \mathrm{kg}^{-1}\right)$ e conteúdos $\left(\mathrm{g}\right.$ planta $\left.{ }^{-1}\right)$ de macronutrientes por classe de sítio, em cada um dos compartimentos da biomassa aérea.

TABELA 2 - Concentração e conteúdo de macronutrientes por classe de sítio ${ }^{1}$ em cada um dos compartimentos da biomassa aérea de bracatinga na região metropolitana de Curitiba.

\begin{tabular}{|c|c|c|c|c|c|c|c|c|c|c|}
\hline \multirow{2}{*}{ Sítio } & \multicolumn{2}{|c|}{$\mathrm{N}$} & \multicolumn{2}{|c|}{$P$} & \multicolumn{2}{|c|}{$\mathrm{K}$} & \multicolumn{2}{|c|}{$\mathrm{Ca}$} & \multicolumn{2}{|c|}{$\mathrm{Mg}$} \\
\hline & $\mathrm{g} \mathrm{kg}^{-1}$ & $\mathrm{~g} \mathrm{pl}^{-1}$ & $\mathrm{~g} \mathrm{~kg}^{-1}$ & $\mathrm{~g} \mathrm{pl}^{-1}$ & $\mathrm{~g} \mathrm{~kg}^{-1}$ & $\mathrm{~g} \mathrm{pl}^{-1}$ & $\mathrm{~g} \mathrm{~kg}^{-1}$ & $\mathrm{~g} \mathrm{pl}^{-1}$ & $\mathrm{~g} \mathrm{~kg}^{-1}$ & $\mathrm{~g} \mathrm{pl}^{-1}$ \\
\hline \multicolumn{11}{|c|}{ Folhas } \\
\hline 1 & $25,89 \mathrm{~ns}$ & $78,76 \mathrm{~ns}$ & $1,75 a$ & $4,85 \mathrm{~ns}$ & $5,70 a$ & $15,76 \mathrm{~ns}$ & $8,52 \mathrm{~ns}$ & $18,66 \mathrm{~ns}$ & $2,39 \mathrm{~ns}$ & $5,71 \mathrm{~ns}$ \\
\hline II & $26,07 \mathrm{~ns}$ & $53,79 n s$ & $1,53 a b$ & $3,17 \mathrm{~ns}$ & $4,95 a b$ & $9,98 \mathrm{~ns}$ & $7,21 \mathrm{~ns}$ & $15,19 \mathrm{~ns}$ & $1,67 \mathrm{~ns}$ & $3,53 \mathrm{~ns}$ \\
\hline III & $24,25 \mathrm{~ns}$ & $76,16 \mathrm{~ns}$ & $1,33 b$ & $4,09 \mathrm{~ns}$ & $4,91 a b$ & $15,76 \mathrm{~ns}$ & $6,09 \mathrm{~ns}$ & $17,62 \mathrm{~ns}$ & $1,51 \mathrm{~ns}$ & $4,13 \mathrm{~ns}$ \\
\hline IV & $23,95 \mathrm{~ns}$ & $43,05 \mathrm{~ns}$ & $1,29 b$ & $2,40 \mathrm{~ns}$ & $4,32 b$ & $7,83 \mathrm{~ns}$ & $4,14 \mathrm{~ns}$ & $6,52 \mathrm{~ns}$ & $1,60 \mathrm{~ns}$ & $2,60 \mathrm{~ns}$ \\
\hline \multicolumn{11}{|c|}{ Galhos $<4 \mathrm{~cm}$} \\
\hline I & $13,48 \mathrm{~ns}$ & $88,72 \mathrm{~ns}$ & $0,31 a$ & $2,03 n s$ & $4,46 n s$ & $30,29 \mathrm{~ns}$ & $6,52 \mathrm{~ns}$ & $41,50 a$ & $0,76 a$ & $5,52 a$ \\
\hline II & $11,83 \mathrm{~ns}$ & $64,41 \mathrm{~ns}$ & $0,27 a b$ & $1,46 \mathrm{~ns}$ & $3,13 \mathrm{~ns}$ & $15,17 \mathrm{~ns}$ & $5,36 \mathrm{~ns}$ & $28,42 a b$ & $0,35 b$ & $1,90 \mathrm{ab}$ \\
\hline III & $13,24 \mathrm{~ns}$ & $69,48 n s$ & $0,23 b$ & $1,23 \mathrm{~ns}$ & $3,35 \mathrm{~ns}$ & $18,04 \mathrm{~ns}$ & $5,33 n s$ & $27,98 a b$ & $0,48 a b$ & $2,30 a b$ \\
\hline IV & $12,63 \mathrm{~ns}$ & $44,41 \mathrm{~ns}$ & $0,24 b$ & $0,81 \mathrm{~ns}$ & $2,28 \mathrm{~ns}$ & $6,95 \mathrm{~ns}$ & $5,47 \mathrm{~ns}$ & $14,80 \mathrm{~b}$ & $0,41 b$ & $1,21 \mathrm{~b}$ \\
\hline \multicolumn{11}{|c|}{ Galhos $\geq 4 \mathrm{~cm}$} \\
\hline I & $9,88 \mathrm{~ns}$ & $105,0 \mathrm{~ns}$ & $0,20 \mathrm{~ns}$ & $1,98 \mathrm{~ns}$ & $4,01 \mathrm{~ns}$ & $34,09 \mathrm{~ns}$ & $3,79 \mathrm{~ns}$ & $42,37 n s$ & $0,54 \mathrm{~ns}$ & $5,83 n s$ \\
\hline II & $8,21 \mathrm{~ns}$ & $47,04 \mathrm{~ns}$ & $0,17 \mathrm{~ns}$ & $1,04 \mathrm{~ns}$ & $3,80 \mathrm{~ns}$ & $19,43 \mathrm{~ns}$ & $3,85 \mathrm{~ns}$ & $23,40 \mathrm{~ns}$ & $0,32 \mathrm{~ns}$ & $2,18 \mathrm{~ns}$ \\
\hline III & $7,13 \mathrm{~ns}$ & $57,37 \mathrm{~ns}$ & $0,13 \mathrm{~ns}$ & $1,01 \mathrm{~ns}$ & $4,08 \mathrm{~ns}$ & $28,25 \mathrm{~ns}$ & $4,09 \mathrm{~ns}$ & $29,53 \mathrm{~ns}$ & $0,45 \mathrm{~ns}$ & $2,76 \mathrm{~ns}$ \\
\hline IV & $8,39 \mathrm{~ns}$ & $21,09 \mathrm{~ns}$ & $0,19 \mathrm{~ns}$ & $0,48 \mathrm{~ns}$ & $2,39 \mathrm{~ns}$ & $6,07 \mathrm{~ns}$ & $2,59 \mathrm{~ns}$ & $6,72 \mathrm{~ns}$ & $0,33 \mathrm{~ns}$ & $0,73 n s$ \\
\hline \multicolumn{11}{|c|}{ Madeira } \\
\hline I & $1,86 \mathrm{~ns}$ & $104,79 a$ & $0,10 \mathrm{~ns}$ & $5,44 a$ & $2,80 \mathrm{~ns}$ & $163,45 a$ & $1,25 \mathrm{~ns}$ & $59,69 a$ & $0,27 \mathrm{~ns}$ & $15,36 a$ \\
\hline II & $2,06 \mathrm{~ns}$ & $74,63 a b$ & $0,09 \mathrm{~ns}$ & $3,30 a$ & $2,54 \mathrm{~ns}$ & $85,75 a$ & $1,02 \mathrm{~ns}$ & $36,72 a b$ & $0,17 \mathrm{~ns}$ & $7,56 a b$ \\
\hline III & $2,32 \mathrm{~ns}$ & $90,42 a$ & $0,08 \mathrm{~ns}$ & $3,11 a b$ & $2,80 \mathrm{~ns}$ & $122,41 a$ & $1,09 \mathrm{~ns}$ & $48,19 a$ & $0,18 \mathrm{~ns}$ & $5,67 a b$ \\
\hline IV & $1,95 \mathrm{~ns}$ & $31,36 b$ & $0,09 \mathrm{~ns}$ & $1,48 b$ & $1,79 \mathrm{~ns}$ & $27,83 b$ & $0,93 \mathrm{~ns}$ & $14,86 b$ & $0,19 \mathrm{~ns}$ & $2,91 b$ \\
\hline \multicolumn{11}{|c|}{ Casca } \\
\hline I & $15,35 \mathrm{~ns}$ & $114,16 a$ & $0,34 a b$ & $2,55 a$ & $6,57 a$ & $49,20 \mathrm{~ns}$ & $12,84 \mathrm{~ns}$ & $88,96 a$ & $0,64 \mathrm{~ns}$ & $4,39 \mathrm{~ns}$ \\
\hline II & $14,37 \mathrm{~ns}$ & $84,65 a b$ & $0,37 a$ & $2,03 a$ & $4,72 a b$ & $23,19 \mathrm{~ns}$ & $13,83 \mathrm{~ns}$ & $74,91 a$ & $0,47 \mathrm{~ns}$ & $2,69 \mathrm{~ns}$ \\
\hline III & $15,95 \mathrm{~ns}$ & $104,34 a$ & $0,27 b$ & $1,65 a$ & $4,71 a b$ & $29,45 \mathrm{~ns}$ & $13,05 \mathrm{~ns}$ & $81,12 a$ & $0,74 \mathrm{~ns}$ & $4,23 \mathrm{~ns}$ \\
\hline IV & $15,98 \mathrm{~ns}$ & $38,00 \mathrm{~b}$ & $0,30 a b$ & $0,72 b$ & $3,50 b$ & $8,25 \mathrm{~ns}$ & $10,45 \mathrm{~ns}$ & $24,48 b$ & $0,61 \mathrm{~ns}$ & $1,49 \mathrm{~ns}$ \\
\hline
\end{tabular}

${ }^{1}$ Classificação de sítio realizada de acordo com Machado et al. (1997). Classe de sítio I: 6 árvores; Classe de sítio II: 6 árvores; Classe de sítio III: 7 árvores; e Classe de sítio IV: 6 árvores.

Valores seguidos de mesma letra não apresentam diferenças significativas de acordo com o teste de Tukey $(\alpha=0,05)$ e ns $=$ não significativo. $\mathrm{pl}=$ planta. 
TÉO, S. J. et al. Concentração e conteúdo de macronutrientes...

Com relação às concentrações de macronutrientes, constata-se que o $\mathrm{P}$ e $\mathrm{K}$ nas folhas e na casca apresentaram diferenças significativas entre as classes de sítio. Para os galhos $<4 \mathrm{~cm}$, foram as concentrações de $\mathrm{P}$ e $\mathrm{Mg}$ que se mostraram significativamente diferentes. Todas as demais concentrações de macronutrientes não apresentaram diferenças significativas entre as classes de sítio.

As concentrações de macronutrientes nas quais $o$ teste de Tukey indicou diferenças significativas entre as classes de sítio, diminuíram da melhor classe (sítio I) para a pior classe (sítio IV), exceto para casca.

De acordo com Silveira et al. (2004), os nutrientes nas folhas estão diretamente relacionados com o desenvolvimento e produção da planta. As diferentes classes de sítio, por sua vez, podem ser entendidas como unidades geográficas relativamente homogêneas que resultam em diferentes níveis de produtividade para uma determinada espécie. Analisando o efeito significativo da qualidade de sítio sobre as concentrações de $\mathrm{P}$ e $\mathrm{K}$, pode-se supor que a adubação com esses macronutrientes poderia aumentar a produtividade dos bracatingais nas piores classes de sítio.

Verificaram-se diferenças significativas para os conteúdos de macronutrientes nas diferentes classes de sítio mais freqüentemente do que para as concentrações, principalmente para os macronutrientes nos compartimentos da biomassa do fuste (madeira e casca). Os conteúdos de macronutrientes foram maiores para a classe de sítio I e menores para a classe de sítio IV, nos casos em que houve diferença significativa. Todavia não há uma relação de diminuição dos conteúdos de nutrientes da melhor para a pior classe de sítio.

Santana et al. (1999) encontraram uma grande variação no conteúdo de nutrientes na biomassa do tronco de árvores de diferentes espécies de eucalipto entre sítios, refletindo, de certa forma, diferenças em produção. Os conteúdos de nutrientes no tronco foram maiores nos sítios mais produtivos e nos materiais genéticos melhor adaptados às condições destes, evidenciando a relação entre nutrição e produção de biomassa.

No entanto, nos resultados desta pesquisa freqüentemente os conteúdos de macronutrientes da classe de sítio III foram maiores do que da classe de sítio II. Isso pode ser entendido quando se observam as idades das árvores que compõem a classes de sítio III, a qual apresenta as árvores mais velhas do banco de dados e, geralmente maiores dimensões e biomassa do que a classe de sítio II.

A Tabela 3 ilustra as concentrações $\left(\mathrm{g} \mathrm{kg}^{-1}\right)$ e conteúdos ( $\mathrm{g}$ planta $\left.^{-1}\right)$ de macronutrientes por classe de idade em cada um dos cinco compartimentos da biomassa aérea de bracatinga.

TABELA 3 - Concentração e conteúdo de macronutrientes por classe de idade ${ }^{1}$ em cada um dos compartimentos da biomassa aérea de bracatinga na região metropolitana de Curitiba.

\begin{tabular}{|c|c|c|c|c|c|c|c|c|c|c|}
\hline \multirow{2}{*}{ Idade (ano) } & \multicolumn{2}{|c|}{$\mathrm{N}$} & \multicolumn{2}{|c|}{$\mathrm{P}$} & \multicolumn{2}{|c|}{$\mathrm{K}$} & \multicolumn{2}{|c|}{$\mathrm{Ca}$} & \multicolumn{2}{|c|}{$\mathrm{Mg}$} \\
\hline & $\mathrm{g} \mathrm{kg}^{-1}$ & $\mathrm{~g} \mathrm{pl}^{-1}$ & $\mathrm{~g} \mathrm{~kg}^{-1}$ & $\mathrm{~g} \mathrm{pl}^{-1}$ & $\mathrm{~g} \mathrm{~kg}^{-1}$ & $\mathrm{~g} \mathrm{pl}^{-1}$ & $\mathrm{~g} \mathrm{~kg}^{-1}$ & $\mathrm{~g} \mathrm{pl}^{-1}$ & $\mathrm{~g} \mathrm{~kg}^{-1}$ & $\mathrm{~g} \mathrm{pl}^{-1}$ \\
\hline \multicolumn{11}{|c|}{ Folhas } \\
\hline $6,0-7,9$ & $22,53 \mathrm{~ns}$ & $46,66 n s$ & $1,68 a$ & $3,55 \mathrm{~ns}$ & $5,39 n s$ & $11,29 \mathrm{~ns}$ & $9,04 a$ & $18,87 \mathrm{~ns}$ & $2,30 a$ & $4,77 \mathrm{~ns}$ \\
\hline $8,0-9,9$ & $22,90 \mathrm{~ns}$ & $33,37 \mathrm{~ns}$ & $1,25 b$ & $1,94 \mathrm{~ns}$ & $4,35 \mathrm{~ns}$ & $6,81 \mathrm{~ns}$ & $4,57 b$ & $6,59 \mathrm{~ns}$ & 1,63ab & $2,20 \mathrm{~ns}$ \\
\hline $10,0-11,9$ & $28,69 \mathrm{~ns}$ & $98,03 \mathrm{~ns}$ & $1,51 a b$ & $5,28 \mathrm{~ns}$ & $4,80 \mathrm{~ns}$ & $17,01 \mathrm{~ns}$ & $4,80 b$ & $14,94 \mathrm{~ns}$ & $1,44 b$ & $4,70 \mathrm{~ns}$ \\
\hline$>12,0$ & $28,31 \mathrm{~ns}$ & $91,27 \mathrm{~ns}$ & $1,17 \mathrm{~b}$ & $3,57 \mathrm{~ns}$ & $4,91 \mathrm{~ns}$ & $15,68 \mathrm{~ns}$ & $4,96 \mathrm{~b}$ & $13,60 \mathrm{~ns}$ & $1,19 \mathrm{~b}$ & $3,27 \mathrm{~ns}$ \\
\hline \multicolumn{11}{|c|}{ Galhos $<4 \mathrm{~cm}$} \\
\hline $6,0-7,9$ & $11,86 b$ & $61,42 a b$ & $0,30 a$ & $1,55 a b$ & $3,40 \mathrm{~ns}$ & $16,16 a b$ & $6,01 \mathrm{~ns}$ & $30,44 n s$ & $0,57 \mathrm{~ns}$ & $2,92 \mathrm{~ns}$ \\
\hline $8,0-9,9$ & $12,11 \mathrm{ab}$ & $30,99 b$ & $0,25 b c$ & $0,63 b$ & $2,47 n s$ & $6,39 b$ & $6,09 \mathrm{~ns}$ & $14,35 \mathrm{~ns}$ & $0,42 \mathrm{~ns}$ & $0,96 \mathrm{~ns}$ \\
\hline $10,0-11,9$ & $14,57 a$ & $106,18 a$ & $0,26 a b$ & $1,97 a$ & $3,36 \mathrm{~ns}$ & $27,32 a$ & $4,90 \mathrm{~ns}$ & $37,66 \mathrm{~ns}$ & $0,53 \mathrm{~ns}$ & $4,52 \mathrm{~ns}$ \\
\hline$>12,0$ & $13,44 a b$ & $66,35 a b$ & $0,20 \mathrm{c}$ & $0,98 a b$ & $4,04 \mathrm{~ns}$ & $20,83 a b$ & $5,34 \mathrm{~ns}$ & $25,54 \mathrm{~ns}$ & $0,37 n s$ & $1,70 \mathrm{~ns}$ \\
\hline \multicolumn{11}{|c|}{ Galhos $\geq 4 \mathrm{~cm}$} \\
\hline $6,0-7,9$ & $8,09 a$ & $74,01 \mathrm{~ns}$ & $0,18 \mathrm{~ns}$ & $1,48 \mathrm{~ns}$ & $3,44 \mathrm{~ns}$ & $24,59 \mathrm{~ns}$ & $4,29 \mathrm{~ns}$ & $36,90 \mathrm{~ns}$ & $0,47 n s$ & $4,13 \mathrm{~ns}$ \\
\hline $8,0-9,9$ & $8,42 a$ & $21,52 \mathrm{~ns}$ & $0,19 \mathrm{~ns}$ & $0,49 \mathrm{~ns}$ & $2,46 n s$ & $6,38 \mathrm{~ns}$ & $2,77 \mathrm{~ns}$ & $7,36 \mathrm{~ns}$ & $0,25 \mathrm{~ns}$ & $0,52 \mathrm{~ns}$ \\
\hline $10,0-11,9$ & $10,47 a$ & $73,54 \mathrm{~ns}$ & $0,19 \mathrm{~ns}$ & $1,27 \mathrm{~ns}$ & $4,11 \mathrm{~ns}$ & $26,74 \mathrm{~ns}$ & $3,43 \mathrm{~ns}$ & $27,53 \mathrm{~ns}$ & $0,48 \mathrm{~ns}$ & $3,27 \mathrm{~ns}$ \\
\hline$>12,0$ & $5,31 \mathrm{~b}$ & $30,47 \mathrm{~ns}$ & $0,12 \mathrm{~ns}$ & $0,69 \mathrm{~ns}$ & $4,53 \mathrm{~ns}$ & $27,12 \mathrm{~ns}$ & $3,42 \mathrm{~ns}$ & $16,78 \mathrm{~ns}$ & $0,29 \mathrm{~ns}$ & $1,47 \mathrm{~ns}$ \\
\hline \multicolumn{11}{|c|}{ Madeira } \\
\hline $6,0-7,9$ & $1,83 \mathrm{~ns}$ & $58,98 b c$ & $0,10 \mathrm{~ns}$ & $3,17 a$ & $2,08 a b$ & $62,33 \mathrm{~ns}$ & $1,12 \mathrm{~ns}$ & $36,49 a$ & $0,21 \mathrm{~ns}$ & $6,78 a b$ \\
\hline $8,0-9,9$ & $1,87 \mathrm{~ns}$ & $28,85 \mathrm{c}$ & $0,09 \mathrm{~ns}$ & $1,38 b$ & $1,67 \mathrm{~b}$ & $24,46 \mathrm{~ns}$ & $0,98 \mathrm{~ns}$ & $15,41 a$ & $0,19 \mathrm{~ns}$ & $2,70 b$ \\
\hline $10,0-11,9$ & $2,33 \mathrm{~ns}$ & $127,21 \mathrm{a}$ & $0,10 \mathrm{~ns}$ & $5,30 a$ & $3,06 a b$ & $179,2 \mathrm{~ns}$ & $0,92 \mathrm{~ns}$ & $52,02 a$ & $0,25 \mathrm{~ns}$ & $15,41 a$ \\
\hline$>12,0$ & $2,44 \mathrm{~ns}$ & $100,1 \mathrm{ab}$ & $0,07 \mathrm{~ns}$ & $3,18 a b$ & $3,71 \mathrm{a}$ & $174,6 \mathrm{~ns}$ & $1,29 \mathrm{~ns}$ & $62,72 a$ & $0,12 \mathrm{~ns}$ & $5,23 a b$ \\
\hline \multicolumn{11}{|c|}{ Casca } \\
\hline $6,0-7,9$ & $14,08 b$ & $63,58 b c$ & $0,35 a$ & $1,54 a$ & $5,41 \mathrm{~ns}$ & $22,80 \mathrm{~ns}$ & $14,74 a$ & $65,56 a$ & $0,70 \mathrm{~ns}$ & $2,98 a$ \\
\hline $8,0-9,9$ & $15,96 a b$ & $35,70 c$ & $0,29 a b$ & $0,65 b$ & $3,59 \mathrm{~ns}$ & $8,01 \mathrm{~ns}$ & $10,79 b$ & $24,01 b$ & $0,54 \mathrm{~ns}$ & $1,19 b$ \\
\hline $10,0-11,9$ & $17,07 a$ & $145,88 a$ & $0,35 a$ & $3,00 a$ & $4,96 n s$ & $46,11 \mathrm{~ns}$ & $10,99 b$ & $96,88 a$ & $0,61 \mathrm{~ns}$ & $4,76 a$ \\
\hline$>12,0$ & $15,72 a b$ & $115,4 a b$ & $0,23 b$ & $1,68 a$ & $4,96 \mathrm{~ns}$ & $36,31 \mathrm{~ns}$ & $11,68 \mathrm{ab}$ & $85,26 a$ & $0,54 \mathrm{~ns}$ & $4,18 a$ \\
\hline
\end{tabular}

${ }^{1}$ Classe de idade 6,0-7,9 anos: 10 árvores; Classe de idade de 8,0-9,9 anos: 5 árvores; Classe de idade de 10,0-11,9 anos: 6 árvores; Classe de idade maior do que 12 anos: 4 árvores.

Valores seguidos de mesma letra não apresentam diferenças significativas de acordo com o teste de Tukey $(\alpha=0,05)$ e ns $=$ não significativo. $\mathrm{pl}=$ planta. 
TÉO, S. J. et al. Concentração e conteúdo de macronutrientes...

As concentrações de $\mathrm{N}$ mostraram diferenças significativas entre as classes de idade para os galhos $<4 \mathrm{~cm}$, galhos $\geq 4 \mathrm{~cm}$ e para casca, contudo sem apresentar indicação clara de aumento ou diminuição com relação às idades.

O P apresentou concentrações significativamente diferentes nos compartimentos da biomassa de folhas, galhos $<4 \mathrm{~cm}$ e casca. Nas folhas e na casca não houve aumento ou diminuição com relação às classes de idade, já para os galhos $<4 \mathrm{~cm}$, é possível identificar que a concentração e $\mathrm{P}$ foi inversamente proporcional à idade.

As concentrações de $\mathrm{Ca}$ e $\mathrm{Mg}$ nas folhas apresentaram os maiores valores para a menor classe de idade, o contrário pode ser observado para o $\mathrm{K}$ na madeira. O Ca na casca, embora tenha apresentado diferenças significativas entre as classes de idade, não apresentou qualquer relação de aumento ou diminuição bem definida. $\mathrm{Na}$ biomassa de folhas, a concentração de $\mathrm{Ca}$ decresce até atingir valores muito próximos das concentrações de K. Conforme Baggio \& Carpanezzi (1997) e Moura et al. (2006), as mudanças de ordem na relação entre concentrações de $\mathrm{Ca}$ e $\mathrm{K}$ são comuns. O procedimento amostral utilizado neste trabalho, sem a discriminação de folhas novas e maduras, pode ter influenciado nos resultados das relações $\mathrm{K} / \mathrm{Ca}$ e, até mesmo, $\mathrm{K} / \mathrm{Mg}$.

Pereira et al. (1984), Ranger et al. (1995) e Laclau et al. (2000), trabalhando com diferentes espécies, encontraram diferenças significativas entre as concentrações de nutrientes em diferentes idades. Esses autores encontraram que, de maneira geral, a concentração de nutrientes foi inversamente proporcional à idade. Por outro lado, Singh (1982) não encontrou diferenças estatisticamente significativas da concentração de nutrientes com relação à idade de árvores de Pinus patula.

Quando os conteúdos de macronutrientes são analisados para as diferentes classes de idade, verifica-se que o $\mathrm{N}$ apresentou diferenças significativas nos galhos $<4 \mathrm{~cm}$, na casca e na madeira. O $\mathrm{P}$ apresentou diferenças significativas nos galhos $<4 \mathrm{~cm}$, na madeira e na casca, e o $\mathrm{K}$ somente nos galhos $<4 \mathrm{~cm}$. Já o Ca e Mg apresentaram diferenças significativas na madeira e na casca. $\mathrm{Na}$ maioria dos casos, os maiores conteúdos de nutrientes ocorreram para a classe de idade de 10 a 11,9 anos, e não houve aumento com relação à idade.

Estudos com diferentes espécies de pinus e eucalipto relataram que, de maneira geral, houve um aumento do conteúdo de nutrientes na biomassa com relação à idade (Singh, 1982; Bellote et al., 1983; Pereira et al., 1984; Laclau et al., 2000).

Entretanto Negi \& Sharma (1984), que analisaram o conteúdo de nutrientes na biomassa de Eucalyptus globulus nas idades de 5 a 16 anos, não encontraram relação direta entre os conteúdos de nutrientes e a idade. Embora a biomassa do fuste das árvores tenha aumentado com a idade, o conteúdo de nutrientes aumentou no início, mas diminuiu nas idades mais avançadas. Assim como ocorreu neste trabalho, Negi \& Sharma (1984) estudaram o conteúdo de nutrientes em um período de tempo após as árvores atingirem o máximo crescimento. Se os nutrientes tivessem sido analisados desde as primeiras idades da árvore até a sua maturidade, uma relação diretamente proporcional entre idade e conteúdo de nutrientes poderia ter sido encontrada.

$\mathrm{Na}$ Tabela 4 são apresentadas as concentrações $\left(\mathrm{g} \mathrm{kg}^{-1}\right)$ e conteúdos $\left(\mathrm{g}\right.$ planta $\left.{ }^{-1}\right)$ de macronutrientes para cada classe diamétrica, em cada um dos compartimentos da biomassa aérea. Não houve diferença significativa para a concentração da maior parte dos macronutrientes em função das classe diamétricas.

Com relação às concentrações de $\mathrm{P}$ e $\mathrm{Ca}$, apenas na madeira apresentaram diferenças significativas entre as classes diamétricas. No caso do $\mathrm{P}$, o teste de "F" da análise de variância indicou diferenças significativas entre as médias, mas o teste de Tukey não. Neste mesmo compartimento, o maior valor de concentrações de Ca corresponde à classe diamétrica intermediária, de 10 a $12,9 \mathrm{~cm}$.

Baggio (1994) analisou as concentrações de nutrientes para diferentes classes de diâmetro à altura do peito para bracatingais da região metropolitana de Curitiba. Assim como nos resultados deste estudo, poucos nutrientes apresentaram diferenças significativas entre as classes diamétricas e, mesmo os que apresentaram diferenças significativas, não mostraram uma distribuição constante ao longo das diferentes classes diamétricas, o que impossibilitou a interpretação do sentido geral da variação. No entanto, Ranger et al. (1995) e Laclau et al. (2000) encontraram tendências de diminuição da concentração de nutrientes com o aumento das dimensões de Pseudotsuga menziesii e eucalipto, respectivamente.

Os conteúdos de todos os macronutrientes apresentaram diferenças significativas entre as classes de diâmetro, sendo que, em todos os compartimentos, os conteúdos foram maiores na maior classe de diâmetro $(>13,0 \mathrm{~cm})$ em relação à classe 7,0 a 9,9 cm. Esse comportamento pode ser explicado pela estreita relação entre o diâmetro e a biomassa, a qual influencia diretamente no conteúdo de nutrientes.

Poder-se-ia esperar que o diâmetro exercesse um efeito com maior significância estatística sobre os conteúdos de nutrientes para os compartimentos da biomassa do fuste (madeira e casca). Esse efeito pode ser observado para $\mathrm{N} \mathrm{e} \mathrm{Ca}$ na casca e para $\mathrm{K}$ e Ca para galhos $\geq 4 \mathrm{~cm}$, em que o teste de Tukey indicou diferenças estatísticas entre as três classes diamétricas. O diâmetro da árvore é uma variável cumulativa, assim como a biomassa do tronco, todavia esse não é o comportamento da biomassa de folhas, galhos finos e raízes finas, que tende a ficar estável com o passar do tempo (Ranger \& Gelhaye, 2001). 
TÉO, S. J. et al. Concentração e conteúdo de macronutrientes...

TABELA 4 - Concentração e conteúdo de macronutrientes por classe diamétrica ${ }^{1}$ em cada um dos compartimentos da biomassa aérea de bracatinga na região metropolitana de Curitiba.

\begin{tabular}{|c|c|c|c|c|c|c|c|c|c|c|}
\hline \multirow{2}{*}{$\begin{array}{c}\text { Diâmetro } \\
(\mathrm{cm})\end{array}$} & \multicolumn{2}{|c|}{$\mathrm{N}$} & \multicolumn{2}{|c|}{$\mathrm{P}$} & \multicolumn{2}{|c|}{$\mathrm{K}$} & \multicolumn{2}{|c|}{$\mathrm{Ca}$} & \multicolumn{2}{|c|}{$\mathrm{Mg}$} \\
\hline & $\mathrm{g} \mathrm{kg}^{-1}$ & $\mathrm{~g} \mathrm{pl}^{-1}$ & $\mathrm{~g} \mathrm{~kg}^{-1}$ & $\mathrm{~g} \mathrm{pl}^{-1}$ & $\mathrm{~g} \mathrm{~kg}^{-1}$ & $\mathrm{~g} \mathrm{pl}^{-1}$ & $\mathrm{~g} \mathrm{~kg}^{-1}$ & $\mathrm{~g} \mathrm{pl}^{-1}$ & $\mathrm{~g} \mathrm{~kg}^{-1}$ & $\mathrm{~g} \mathrm{pl}^{-1}$ \\
\hline \multicolumn{11}{|c|}{ Folhas } \\
\hline $7,0-9,9$ & $23,62 \mathrm{~ns}$ & $34,33 b$ & $1,38 \mathrm{~ns}$ & $1,97 b$ & $4,50 \mathrm{~ns}$ & $6,42 b$ & $5,97 \mathrm{~ns}$ & $6,94 b$ & $1,76 \mathrm{~ns}$ & $2,20 b$ \\
\hline $10,0-12,9$ & $26,00 \mathrm{~ns}$ & $46,45 b$ & $1,56 \mathrm{~ns}$ & $2,61 b$ & $5,38 \mathrm{~ns}$ & $8,96 b$ & $7,14 \mathrm{~ns}$ & $11,92 b$ & $1,84 \mathrm{~ns}$ & $3,00 b$ \\
\hline$>13,0$ & $25,87 \mathrm{~ns}$ & $114,79 a$ & $1,51 \mathrm{~ns}$ & $6,65 a$ & $5,19 \mathrm{~ns}$ & $23,11 a$ & $6,53 \mathrm{~ns}$ & $26,60 a$ & $1,76 \mathrm{~ns}$ & $7,12 \mathrm{a}$ \\
\hline \multicolumn{11}{|c|}{ Galhos $<4 \mathrm{~cm}$} \\
\hline $7,0-9,9$ & $12,51 \mathrm{~ns}$ & $38,82 b$ & $0,26 \mathrm{~ns}$ & $0,77 b$ & $2,80 \mathrm{~ns}$ & $7,85 b$ & $5,92 \mathrm{~ns}$ & $15,81 b$ & $0,45 \mathrm{~ns}$ & $1,19 b$ \\
\hline $10,0-12,9$ & $13,31 \mathrm{~ns}$ & $70,23 a b$ & $0,27 \mathrm{~ns}$ & $1,41 a$ & $4,14 \mathrm{~ns}$ & $20,65 a$ & $5,76 \mathrm{~ns}$ & $29,75 a$ & $0,53 \mathrm{~ns}$ & $2,68 a b$ \\
\hline$>13,0$ & $12,75 \mathrm{~ns}$ & $98,97 a$ & $0,26 \mathrm{~ns}$ & $2,09 a$ & $3,22 \mathrm{~ns}$ & $27,22 a$ & $5,23 \mathrm{~ns}$ & $42,24 a$ & $0,53 \mathrm{~ns}$ & $4,66 a$ \\
\hline \multicolumn{11}{|c|}{ Galhos $\geq 4 \mathrm{~cm}$} \\
\hline $7,0-9,9$ & $7,64 \mathrm{~ns}$ & $12,48 b$ & $0,17 n s$ & $0,28 b$ & $3,32 \mathrm{~ns}$ & $4,27 \mathrm{c}$ & $3,46 n s$ & $4,63 c$ & $0,46 n s$ & $0,54 b$ \\
\hline $10,0-12,9$ & $8,92 \mathrm{~ns}$ & $31,61 b$ & $0,18 \mathrm{~ns}$ & $0,68 b$ & $4,28 \mathrm{~ns}$ & $13,46 b$ & $4,19 \mathrm{~ns}$ & $15,22 b$ & $0,37 n s$ & $1,40 \mathrm{~b}$ \\
\hline$>13,0$ & $8,18 \mathrm{~ns}$ & $122,01 a$ & $0,16 \mathrm{~ns}$ & $2,28 a$ & $3,59 \mathrm{~ns}$ & $47,89 a$ & $3,53 \mathrm{~ns}$ & $55,60 a$ & $0,42 \mathrm{~ns}$ & $6,34 a$ \\
\hline \multicolumn{11}{|c|}{ Madeira } \\
\hline $7,0-9,9$ & $1,99 \mathrm{~ns}$ & $33,80 \mathrm{~b}$ & $0,10 a$ & $1,63 b$ & $2,18 \mathrm{~ns}$ & $38,18 b$ & $0,94 b$ & $16,29 b$ & $0,19 \mathrm{~ns}$ & $2,81 b$ \\
\hline $10,0-12,9$ & $2,29 \mathrm{~ns}$ & $77,13 a$ & $0,10 a$ & $3,71 a$ & $3,18 \mathrm{~ns}$ & $111,86 a$ & $1,41 a$ & $48,04 a$ & $0,24 \mathrm{~ns}$ & $8,68 a$ \\
\hline$>13,0$ & $1,93 \mathrm{~ns}$ & $127,45 a$ & $0,08 a$ & $5,11 \mathrm{a}$ & $2,29 \mathrm{~ns}$ & $169,29 a$ & $0,94 b$ & $63,22 a$ & $0,19 \mathrm{~ns}$ & $13,23 a$ \\
\hline \multicolumn{11}{|c|}{ Casca } \\
\hline $7,0-9,9$ & $15,18 \mathrm{~ns}$ & $40,96 c$ & $0,31 \mathrm{~ns}$ & $0,85 b$ & $4,54 \mathrm{~ns}$ & $12,69 b$ & $11,71 \mathrm{~ns}$ & $32,10 c$ & $0,66 \mathrm{~ns}$ & $1,71 b$ \\
\hline $10,0-12,9$ & $14,59 \mathrm{~ns}$ & $82,04 b$ & $0,33 \mathrm{~ns}$ & $1,81 a$ & $5,76 \mathrm{~ns}$ & $32,35 a$ & $12,74 \mathrm{~ns}$ & $68,35 b$ & $0,54 \mathrm{~ns}$ & $2,93 b$ \\
\hline$>13,0$ & $16,49 \mathrm{~ns}$ & $145,92 a$ & $0,32 \mathrm{~ns}$ & $2,77 a$ & $4,50 \mathrm{~ns}$ & $42,07 a$ & $13,48 \mathrm{~ns}$ & $112,31 a$ & $0,64 \mathrm{~ns}$ & $5,43 a$ \\
\hline
\end{tabular}

${ }^{1}$ Classe diamétrica menor do que $10 \mathrm{~cm}$ : 10 árvores; Classe diamétrica de 10,0-12,9 cm: 7 árvores; Classe diamétrica maior do que $13 \mathrm{~cm}$ : 8 árvores.

Valores seguidos de mesma letra não apresentam diferenças significativas de acordo com o teste de Tukey $(\alpha=0,05)$ e ns $=$ não significativo. $\mathrm{pl}=$ planta.

\section{CONCLUSÕES}

1) As concentrações de macronutrientes na biomassa aérea de bracatinga não apresentaram relação direta com as classes de idade e diâmetro.

2) As concentrações de $P$ e $K$ nas folhas diminuíram da melhor para a pior classe de sítio dos bracatingais.
3) Não foram observadas relações de aumento ou diminuição entre os conteúdos de macronutrientes da biomassa de bracatinga e as diferentes classes de sítio e idade.

4) Os conteúdos de macronutrientes foram diretamente proporcionais aos diâmetros das árvores.

\section{REFERÊNCIAS}

1. BAGGIO, A. J. Estudio sobre el sistema agroforestal tradicional de la bracatinga (Mimosa scabrella Benth.) en Brasil: productividad, manejo de residuos y elaboracion de compost. 242 f. Tese (Doutorado) - Universidad Politecnica de Madrid, Madrid, 1994.

2. BAGGIO, A. J.; CARPANEZZI, A. A. Exportação de nutrientes na exploração de bracatingais. Boletim de Pesquisa Florestal, n. 34, p. 3-15. 1997.

3. BELLOTE, A. F. J.; SARRUGE, J. R.; HAAG, H. P. OLIVEIRA, G. D. de. Absorção de macronutrientes e micronutrientes pelo Eucalyptus grandis (Hill, ex-Maiden) em função da idade. Silvicultura, n. 32, p. 633-643, 1983.

4. CALDEIRA, M. V. W.; SCHUMACHER, M. V.; SANTOS, E. M. dos.; TEDESCO, N.; PEREIRA, J. C. Estimativa do conteúdo de nutrientes em um povoamento jovem de Acacia mearnsii De Wild. estabelecido na região sul do Brasil. Floresta, v. 29, n. 1, p. 53-65, 2000a.

5. CALDEIRA, M. V. W.; SCHUMACHER, M. V.; TEDESCO, N.; SANTOS, E. M. dos. Ciclagem de nutrientes em Acacia mearnsii De Wild. V. quantificação do conteúdo de nutrientes na biomassa aérea Acacia mearnsii De Wild. procedência australiana. Ciência Rural, v. 30, n. 6, p. 977-982, 2000b.

6. CALDEIRA, M. V. W.; SCHUMACHER, M. V.; SANTOS, E. M. dos. Conteúdo de nutrientes em uma procedência de Acacia mearnsii plantada no Rio Grande do Sul - Brasil. Boletim de Pesquisa Florestal, n. 42, p. 105-121, 2001.

7. CALDEIRA, M. V. W.; RONDON NETO, R. M.; SCHUMACHER, M. V.; WATZLAVICK, L. F. Exportação de nutrientes em função do tipo de exploração em um povoamento de Acacia mearnsii De Wild. Floresta e Ambiente, v. 9, n. 1, p. 97104, 2002. 
TÉO, S. J. et al. Concentração e conteúdo de macronutrientes...

8. EPSTEIN, E.; BLOOM, A. Nutrição mineral de plantas: princípios e perspectivas. 2 ed. Londrina: Editora Planta, 2006. $401 \mathrm{p}$.

9. FABROWSKI, F. J.; MUÑIZ, G. I. B. de; MAZZA, M. C. M.; NAKASHIMA, T.; KLOCK, U.; POSSAMAI, J. C.; NISGOSKI, $\mathrm{S}$. Anatomia comparativa da madeira das variedades populares de bracatinga (Mimosa scabrella Bentham). Ciência Florestal, v. 15, n. 1, p. 65-73, 2005.

10. LACLAU, J. P.; BOUILLET, J. P.; RANGER, J. Dynamics of biomass and nutrient accumulation in a clonal plantation of Eucalyptus in Congo. Forest Ecology and Management, v. 128, n. 3, p. 181-196, 2000.

11. MAACK, R. Geografia física do estado do Paraná. 3. ed. Curitiba: Imprensa Oficial do Paraná, 2002. $440 \mathrm{p}$.

12. MACHADO, S. A.; OLIVEIRA, E. B.; CARPANEZZI, A. A.; BARTOSZECK, A. C. P. S. Classificação de sítio para bracatingais na região metropolitana de Curitiba. Boletim de Pesquisa Florestal, n. 35, p. 21-37, 1997.

13. MARTINS, A. P. L.; REISSMANN, C. B. Material vegetal e as rotinas laboratoriais nos procedimentos químico-analíticos. Scientia Agraria, v. 8, n. 1, p. 1-17, 2007.

14. MOURA, O. N.; PASSOS, M. A. A.; FERREIRA, R. L. C.; MOLICA, S. G.; LIRA JUNIOR, M. A.; LIRA, M. A.; SANTOS M. V. F. dos. Distribuição de biomassa e nutrientes na parte aérea de Mimosa caesalpiniaefolia Benth. Revista Árvore v. 30 , n. 6 , p. 877-884, 2006.

15. NAVARRO, L. A.; DUARTE, L.; KLEIN, R. C.; HEUSER, E. D. Caracterização dos cristais de oxalato de cálcio nas partes reprodutivas e vegetativas aéreas de llex paraguariensis A. St. Hil. (Aquifoliaceae). Revista Brasileira de Biociências, v. 5, supl. 1, p. 762-764, 2007.

16. NEGI, J. D. S.; SHARMA, D. C. Distribution of nutrient in an age series of Eucalyptus globulus plantation in Tamil Nadu. Indian Forester, v. 110, n. 9, p. 944-953, 1984.

17. PEREIRA, A. R.; BARROS, N. F. de; ANDRADE, D. C. de; CAMPOS, P. T. A. Concentração e distribuição de nutrientes em Eucalyptus grandis em função da idade cultivado na região do cerrado. Brasil Florestal, n. 59, p. 27-37, 1984

18. PERRY, D. A. Forest ecosystems. Baltimore: The Johns Hopkins University Press, 1994. 649 p.

19. PRATA, R. R.; MENDONÇA, M. S. de. Estudo anatômico do xilema secundário da raiz e do caule de Maytenus guyanensis Klotzsch ex Reissek (Celastraceae). Acta Amazonica, v. 39, n. 2, p. 261-266, 2009.

20. RANGER, J.; GELHAYE, D. Belowground biomass and nutrient content in a 47-year-old Douglas-fir plantation. Annals of Forest Science, v. 58, n. 4, p. 423-430, 2001.

21. RANGER, J.; MARQUES, R.; COLIN-BELGRAND, M.; FLAMMANG, N.; GELHAYE, D. The dynamics of biomass and nutrient accumulation in a Douglas-fir (Pseudotsuga menziesii Franco) stand studied using a chronosequence approach Forest Ecology and Management, v. 72, n. 2, p. 167-183, 1995.

22. SANTANA, R. C.; BARROS, N. F. de; NEVES, J. C. L. Biomassa e conteúdo de nutrientes de procedências de Eucalyptus grandis e Eucalyptus saligna em alguns sítios florestais do estado de São Paulo. Scientia Forestalis, n. 56, p. 155-169. 1999

23. SILVEIRA, L. V. de A.; HIGASHI, E. N.; GONÇALVES, A. N.; MOREIRA, A. Evaluation of the nutritional status of eucalypts: visual and foliar diagnoses and their interpretation. In: GONÇALVES, J. L. de M.; BENEDETTI, V. Forest nutrition and fertilization. Piracicaba: IPEF, 2004. p. 13-64.

24. SINGH, B. Nutrient content of standing crop and biological cycling in Pinus patula ecosystem. Forest Ecology and Management, v. 4, n. 4, p. 317-332, 1982.

25. SCHUMACHER, M. V.; CALDEIRA, M. V. W.; Estimativa da biomassa e do conteúdo de nutrientes de um povoamento de Eucalyptus globulus (Labillardière) sub-espécie maidenii. Ciência Florestal, v. 11, n. 1, p. 45-53, 2001

26. SCHUMACHER, M. V.; BRUN, E. J.; ILLANA, V. B.; DISSIUTA, S. I.; AGNE, T. L. Biomassa e nutrientes em um povoamento de Hovenia dulcis Thunb., plantado na Fepagro Florestas, Santa Maria, RS. Ciência Florestal, v. 18, n. 1, p. 27-37, 2008.

27. SOMARRIBA, E.; KASS, D. Estimates of above-ground biomass and nutrient accumulation in Mimosa scabrella fallows in southern Brazil. Agroforestry Systems, v. 51, n. 2, p. 77-84, 2001

Recebido em 29/10/2009 Aceito em 22/11/2010 
